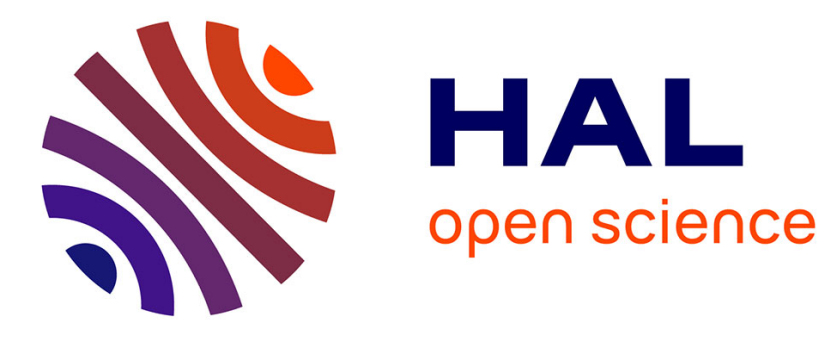

\title{
Routing impact on Network Lifetime Maximization using Power/Rate trade-off in WVSN
}

Nesrine Khernane, Jean-François Couchot, Ahmed Mostefaoui

\section{To cite this version:}

Nesrine Khernane, Jean-François Couchot, Ahmed Mostefaoui. Routing impact on Network Lifetime Maximization using Power/Rate trade-off in WVSN. International Wireless Communications and Mobile Computing Conference, Jun 2017, Valencia, Spain. hal-02963392

\section{HAL Id: hal-02963392 \\ https://hal.science/hal-02963392}

Submitted on 10 Oct 2020

HAL is a multi-disciplinary open access archive for the deposit and dissemination of scientific research documents, whether they are published or not. The documents may come from teaching and research institutions in France or abroad, or from public or private research centers.
L'archive ouverte pluridisciplinaire HAL, est destinée au dépôt et à la diffusion de documents scientifiques de niveau recherche, publiés ou non, émanant des établissements d'enseignement et de recherche français ou étrangers, des laboratoires publics ou privés. 


\section{Routing impact on Network Lifetime Maximization using Power/Rate trade-off in WVSN}

\author{
Nesrine KHERNANE \\ FEMTO-ST Institute, CNRS, \\ Univ. Bourgogne Franche-Comte \\ UBFC, Belfort, France \\ nesrine.khernane@univ-fcomte.fr
}

\author{
Jean-François Couchot \\ FEMTO-ST Institute, CNRS, \\ Univ. Bourgogne Franche-Comte \\ UBFC, Belfort, France \\ jean-francois.couchot@univ-fcomte.fr
}

\author{
Ahmed Mostefaoui \\ FEMTO-ST Institute, CNRS, \\ Univ. Bourgogne Franche-Comte \\ UBFC, Belfort, France \\ ahmed.mostefaoui@univ-fcomte.fr
}

\begin{abstract}
In Wireless multimedia sensor networks (WMSNs), one of the most important issues is to balance video data coding versus the perceived video quality at the sink, while maximizing the network lifetime. That is, better quality generates higher data rates and consequently consumes additional resources (i.e. energy). Finding such a balance is not obvious, especially in a distributed fashion. Previous research works have tackled this issue by not considering the routing issue; i.e. they consider that it has been done separately during the network initialization. In other term, routing is "viewed" as a network input and not as a parameter to optimize. The purpose of this paper is to demonstrate, through extensive simulations, the impact of the routing policy on the approaches used to find such a balance. To this end, we first considered several well-known routing approaches in WMSNs and extensively analyzed the impact of each one by considering three main parameters: battery consumption, activity duration, and network lifetime. The simulation results show clearly the great impact of the considered routing approaches on the obtained resource's consumption balance.
\end{abstract}

Index terms- WMSN, network lifetime, distributed, routing, optimize

\section{INTRODUCTION}

Different from traditional sensor networks (WSNs), where data processing is usually simple and even negligible in terms of energy consumption, in WMSNs, the captured multimedia content usually needs to be coded/processed before transmission. This is particularly true in some applications as target tracking, video surveillance, etc., where sensors are supposed to handle high data rates to provide the desired video quality. Consequently, additional resources are consumed (processing and transmission), which can greatly limit the performance of the network in term of operational lifetime.

The research issue is then to find a balance between the visual quality at the sink and the video coding at the source nodes, since higher quality generates higher data rates and consequently consumes additional resources. Nevertheless, previous approaches [7], [5], also called power-rate-distortion approaches, have not considered the routing issue and the latter has been left as a separate issue. In other word, the routing is supposed to be done/fixed during the initialization of the network and hence is considered after as an input (i.e. predefined) in the optimization process.

We argue that maximizing network lifetime depends not only on power-rate-distortion optimization, but also on the chosen routing protocol across the network by optimally selecting the forwarding nodes. To the best of our knowledge, the only work that considers the last two parameters was described in [12]. However, the authors propose a centralized approach that intrinsically limits its applicability (i.e. weaknesses of centralized approach within large distributed sensor networks).

In a previous work [10], we proposed a distributed approach based on a power-rate-distortion model [8] which ensures a trade-off between the desirable visual quality at the sink and the available network's resources, mainly encoding power and data rates, in order to prolong the network lifetime. Based on this approach, we aim, within this paper, to evaluate the impact of routing protocols on network lifetime in WMSNs. We analyze thus the behavior of various routing protocols implemented as an add-on to the fully distributed algorithm of our previous work. The analysis was conducted on several configurations studying the following parameters: convergence delay, network lifetime improvement and battery consumption of these different routing algorithms.

This paper is organized as follows: Section II presents the related work. In Section III we describe the system model. In Section IV we describe a set of routing protocols which have been implemented in this work. In Section V we compare and evaluate the impact on network lifetime of these routing protocols. Results are presented in section VI. Concluding remarks and future work are finally outlined in VII.

\section{RELATED WORK}

WMSN is a special type of wireless sensor networks (WSNs), that can wirelessly communicate scalar data as well as multimedia data (e.g. audio, image, and video). If not properly routed, this latter dramatically decreases both the desired video quality and the network lifetime. In the past few years, many research works on WMSN routing algorithms. The intent behind the proposed solutions is to improve the performance of WMSNs in terms of quality of service and energy conservation in order to maximize the network lifetime.

Based on the hierarchical structure of the network, Bi et al. [2] have proposed an ant-colony routing algorithm for WMSNs. In order to meet: delay, packet-loss, energy and memory requirements, authors only address the routing scheme between the cluster heads and the sink. To reach the 
sink, paths over the multi-hop communication are selected with respect to the aforementioned QoS metrics on each path. However, control packets used in this solution for route discovery cause a high overhead. Each sensor node periodically broadcasts a HELLO packet and forwards ants that have to reach the sink and go back for route discovery.

Focusing on energy constraint, authors in [13] have proposed a solution (EEQAR), in which the WMSN routing is modeled by communications in a social network. EEQAR adopts a cluster hierarchy to meet flexibility and communication efficiency. However, the authors made a strong assumption that the cluster heads ( $\mathrm{CHs}$ ) are not limited by energy. In addition, we notice that first an additional overhead is required for route discovery inside the cluster. Furthermore, the evaluation of the video quality level has not been considered.

To address this problem, a Multi-hop hierarchical routing protocol for Efficient VIdeo communication (MEVI) [3] have been proposed. Composed by both scalar and camera nodes (denoted as CHs). MEVI, first selects the $\mathrm{CHs}$ based on the Link Quality Indicator. Then, it uses two types of messages for route discovery (i.e., route request and route reply). The base station can also send requests to the $\mathrm{CH}$, that will turn its FoV to the desired location and transmits the video content to the BS. However, a failure of one $\mathrm{CH}$ may cause the network to lose a part of its tracking area, and may cause the network to partition into disjoint blocks.

Different from the aforementioned clustering-based routing protocols, Delay and Link Utilization Aware Routing Protocol [6] was proposed for WMSNs. Three metrics were considered in routing decision: packet service time, channel utilization and remaining energy. The proposed protocol, selects the forwarding nodes by enabling the communication between network and MAC layers, in order to minimize endto-end delay while ensuring a minimal distance as possible from source node to destination. However, the simulation results in terms of average throughput and end-to-end delay show that more is the number of nodes, less is the performance of this protocol compared to previous protocols.

In [14], authors have proposed a new multi-objective approach for the WMSN routing problem. The Expected Transmission Count (ETX) and delay are used as QoS parameters in order to produce a diverse set of optimal solutions. The selection of the first path from source to destination (called initial population) is implemented with a Breadth First Search(BFS) algorithm. Since the execution time of the latter depends on the number of nodes, authors have used a crossover and mutation genetic operators to find the feasible intermediate sensor nodes. However, the energy consumed by each node during routes discovery have not been considered.

Nonetheless, the aforementioned routing protocols did not consider at all the encoding power at each node, which can affect not only the desired video quality, but also the network lifetime. On the other hand, getting the system through two optimization phases (i.e. routing then power-rate-distortion optimizations) will undoubtedly tumble down the network.

To the best of our knowledge, the only work that considered both: a) power-rate-distortion optimization, b) and routing discovery in WMSNs, for network lifetime maximization is described in [12]. The proposed algorithm assumed that a set of video sensor nodes are woken up only during data transmission period. Then, it selected a set of paths, that join every active node to the sink. However, the optimization problem does not consider the reception power consumption that have a direct impact on network lifetime. Additionally, the routing and channel allocation information are monitored by the sink (i.e., in a centralized manner), and must be updated at nodes. It results an additional power consumption for transmission and reception of such an information.

\section{SYSTEM MODEL}

In this section, we briefly review: network model, problem formulation and resolution of our previous fully distributed solution, designed to ensure an optimal trade-off between encoding power and data rate, under a pre-defined routing matrix assumption. More details can be found in [10].

\section{A. Network model}

We consider a network composed of a set $N$ of potential video sensor nodes and a collector point (i.e. the sink). Each video sensor $h \in N$ transmits both: its own data, and all the incoming traffic from its neighbors to the sink.

Under the unidirectional oriented graph assumption, let $a_{i l}$ denote the matrix of link information, in which 1 presents an outgoing traffic from $i,-1$ presents an incoming traffic to $i$, and 0 otherwise. Moreover, the outgoing links (resp. with incoming links) are represented with $a_{i l}^{+}$matrix (resp. with $a_{i l}^{-}$matrix), whose elements are defined by: 1 if a given link is an outgoing link from $i$ (resp. with an incoming link) and 0 otherwise. Each video sensor $h$ generates data traffic with source rate $R_{h}$ after capturing and encoding a given video, and transmits this latter with respect to the link rate $x_{h l}$. Thus, the flow conservation (denoted $\eta_{h i}$ ) at each node can be formulated as follows:

$$
\sum_{l \in \mathcal{L}} a_{i l} x_{h l}=\eta_{h i}= \begin{cases}R_{h} & \text { if } i \text { is the generator of traffic } \\ -R_{h} & \text { if } i \text { is the sink } \\ 0 & \text { otherwise. }\end{cases}
$$

\section{B. Background}

Prolong the operational lifetime of a WVSN is one of the most critical issues. In a such network, we have indeed to consider not only the energy consumption during data communication, but also the energy consumption during video compression that can be defined as the following:

Video coding $\left(P_{s h}\right)$ : We used the power-rate-distortion $(\mathrm{P}-$ R-D) analytical model presented in [7], [9]

$$
P_{s h}=\left(\frac{\ln \left(\sigma^{2}\right)-\ln \left(D_{h}\right)}{\gamma * R_{h}}\right)^{\frac{2}{3}},
$$

where $\sigma^{2}$ is the average input variance, $D_{h}$ is the encoding distortion, and $\gamma$ is the encoding efficiency coefficient and $R_{h}$ is the source rate. This model, introduces the trade-off between 
the encoding power consumption and the generated rate of each source node, while taking into account the desired video quality at the sink.

Transmission $\left(P_{t i}\right)$ : the power consumption model for data transmission at node $i$ is:

$$
P_{t i}=\sum_{l \in \mathcal{L}} a_{i l}^{+} *\left(\alpha+\beta d_{l}^{n_{p}}\right) * y_{l},
$$

where $\alpha$ and $\beta$ are transmit electronics parameters, $d_{l}$ is the distance between the transmitter and the receiver, $n_{p}$ is the path-loss exponent and $y_{l}$ corresponds to the aggregate rate transmitted through link $l$.

Reception $\left(P_{r i}\right)$ : similarly, the power consumption model for data reception at node $i$ can be formulated as:

$$
P_{r i}=c^{r} * \sum_{l \in \mathcal{L}} a_{i l}^{-} * y_{l}
$$

where $c^{r}$ is the radio receiver energy consumption cost.

In this paper, we consider critical applications in which the energy depletion of the first node leads to the death of the whole network, defined as:

$$
T_{n e t}=\min _{i \in N} B_{i} / P_{i},
$$

where $T_{\text {net }}$ is the network lifetime, $B_{i}$ is the initial energy of node $i$ and $P_{i}=P_{s h}+P_{t i}+P_{r i}$ is the total energy consumption of node $i$.

\section{Problem formulation and resolution}

Note that, in this subsection we assume that the routing is completely pre-defined (i.e. will not be integrated in the optimization steps), and justly, the objective of this paper is to analyze the impact of routing on the maximum achievable network lifetime. And thus, demonstrate the necessity to integrate routing in the optimization problem.

Based on the aforementioned formulas. Let $q=1 / T_{\text {net }}$ be the inverse lifetime of the network, that should be maintained as a common variable. To formulate a fully distributed problem, an auxiliary variable, $q_{i}(\forall i \in N)$, has been introduced at each node $i$, such that: $q_{i}=q_{j}(\forall i, j \in N)$. This condition should be expressed by the: $\sum_{i \in N} a_{i l} . q_{i}=0(\forall l \in \mathcal{L})$ constraint.

To ensure convexity of the optimization problem with respect to $q_{i}, R_{h}, x_{h l}$ and $P_{s h}$, powers should be introduced into the objective function, with some regular factors (namely $\delta_{r}, \delta_{x}$ and $\delta_{p}$ ) in order to keep their impact as minimal as possible on the objective function. The final problem, without routing consideration, can be then formulated as follow:

$$
\begin{aligned}
\underset{\left(R_{h}, x_{h l}, P_{s h}\right)}{\operatorname{minimize}} & \sum_{i \in N} q_{i}^{2}+\delta_{x} \sum_{h, l} x_{h l}^{2}+\delta_{r} \sum_{h} R_{h}^{2}+\delta_{p} \sum_{h} P_{s h}^{8 / 3} \\
\text { subject to } & \sum_{l \in \mathcal{L}} a_{i l} . x_{h l}=\eta_{h i}, \\
& \sigma^{2} e^{\gamma \cdot R_{h} \cdot P_{s h}^{2 / 3}} \leq D_{h}, \\
& P_{i} \leq q_{i} \cdot B_{i}, \\
& \sum_{i \in N} a_{i l} \cdot q_{i}=0, \\
& x_{h l} \geq 0, R_{h} \geq 0, P_{s h}>0, q_{i}>0 .
\end{aligned}
$$

The problem constitutes a convex optimization problem with respect to all decision variables [1], which can be solved by Primal-Dual method [15]. The details of the solution can be found in [10].

\section{ROUTING PROTOCOLS}

Routing is one of the most important design issues in WMSN. Therefore, several metrics should be taking into account in order to ensure an optimal propagation of data, namely, energy consumption, delay, bandwidth, video quality.

In this section, we recall the definition of the different wellknown routing protocols to be used in the comparison via simulations in section $\mathrm{V}$ :

- BroadcastRout: Each node $i$ in the network, except the sink, broadcasts the packet to all its neighboring nodes.

- ProbaRout: Each node $h$ chooses one node among its neighbors in an equitable manner, with a probability $P$ set to $\frac{1}{N b r s_{h}}$, where $N_{b r s}$ denotes the number of $h$ 's neighbors.

- GeomRout: Each node $h$ verifies at first if the sink is in its communication range. In the positive case, it sends the packet directly to the sink. Otherwise, it chooses the closest node to itself in the direction of the destination.

- ShortPathHop: In this routing protocol, each node $h$ selects the shortest path to the sink in term of number of hops. The chosen intermediate sensor node should be the farthest one (in the communication range of $h$ ), among the neighboring nodes of $h$, in the direction of the destination.

- ShortPathDist: This strategy consists of choosing the shortest path to the destination in term of distance (w.r.t. a given distance). Thus, the chosen candidate may be the nearest one, the farthest or even the one in between.

- MultiPathRout: Each node $h$ in the network, selects randomly two of its neighboring nodes to reach the destination. At the end, the sink should be chosen by at least two sensor nodes (those that they have it in the communication range) as one of their paths.

- ArbitraryRout: This strategy is the one defined in our previous work, and heavily borrowed from [7]. The objective was to send data over multi-paths, that were chosen in a completely arbitrary manner.

\section{Simulation Results}

In this section, we evaluate the impact of each of the aforementioned routing protocols, through extensive simulations. The routing protocols were implemented as an add on to the optimization problem presented in section III (i.e. the routes and neighbors were computed separately before starting the optimization steps), through MiXiM framework [11], using OMNET++ simulator [16]. The simulation notations with default values can be found in Table I.

Figure 1 depicts the configuration networks $C_{1}$ and $C_{2}$, respectively.

One of the most critical issues in WMSN is to prolong the network lifetime. For this, it should be known that the 


\begin{tabular}{|c|c|c|}
\hline Notation & Description & Value \\
\hline$\sigma^{2}$ & Variance of video encoder (in terms of MSE) & 3500 \\
\hline$\gamma$ & Encoding efficiency coefficient & $55.54 \mathrm{~W}^{3 / 2} \cdot \mathrm{Mb}^{-1} \cdot \mathrm{s}^{-1}$ \\
\hline$B_{i}$ & Initial energy at each node & $5.0 \mathrm{MJ}$ \\
\hline$\delta_{x}, \delta_{r}, \delta_{p}$ & Regularization factors & 0.2 \\
\hline$\rho$ & Step size parameter & 0.15 \\
\hline$D_{h}$ & Distortion of an encoding frame (in terms of MSE) & 100 \\
\hline$\alpha$ & Energy cost of the transmit electronics & $0.5 \mathrm{~J}^{\mathrm{M}} \mathrm{Mb}^{-1}$ \\
\hline$\beta$ & Coefficient term of the transmit amplifier & $1.3 \times 10^{-8} \mathrm{~J} \cdot \mathrm{Mb}^{-1} \cdot \mathrm{m}^{-4}$ \\
\hline$n_{p}$ & Path loss exponent & 4 \\
\hline$c^{r}$ & Energy consumption cost of radio receiver & $0.5 \mathrm{~J} . \mathrm{Mb}^{-1}$ \\
\hline$p_{s h}$ & Encoding power of Panoptes video sensor nodes [4] & $3.05 \mathrm{~W}$ \\
\hline
\end{tabular}

TABLE I

USED MODEL PARAMETERS WITH THEIR DEFAULT VALUES.
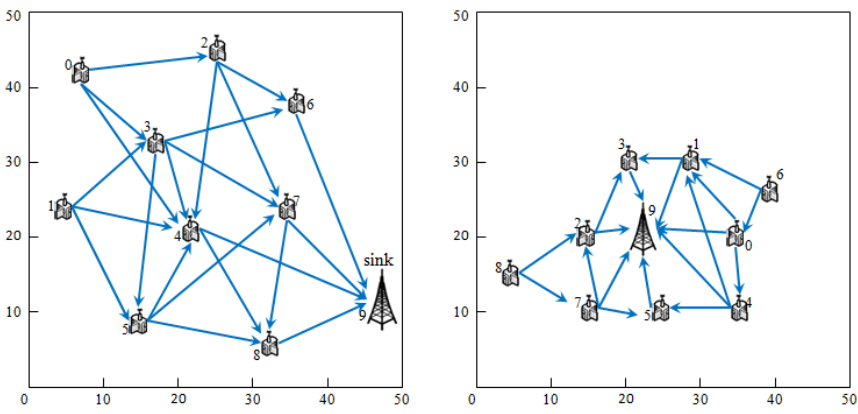

Fig. 1. Network configurations: $C_{1} C_{2}$

two dominant operations that cause the network lifetime breakdown are: data transmission and video coding.

In this section we investigate the impact of data routing on a fully distributed system that provides a compromise between the encoding power and data rate.

First, let us recall that the distributed solution can be satisfied if and only if all the $q_{i}(i \in N)$ converge to a common variable $q$. In this paper, we consider the system as completely stable when the maximum variation between the $q_{i}(i \in N)$ is $10^{-2}$, in order to minimize energy consumption required for a system to gain more precision (with respect to $q_{i}$ ). Three fundamental parameters were studied:

1) Energy cost: starting by comparing the energy cost for each routing protocol. Figure 2 shows the battery consumption of the optimization steps for the two configurations $C_{1}$ and $C_{2}$. For the former configuration (Fig.2(a)), we can see that at the $10^{-2}$ precision the BroadcastRout achieves the lowest battery consumption compared to author protocols, while the ProbaRout protocol presents the highest $(1.215 \%)$ battery consumption. This can be explained by the fact that, even if the BroadcastRout protocol floods the network by packets, it takes much less iteration to converge. For the latter configuration, this figure (Fig.2(b)) shows that the ShortPathHop, ShortPathDist and GeomRout act exactly the same way, and have the lowest battery consumption, while the ProbaRout strategy remains the most demanding in term of battery consumption, regarding the number of iteration required to achieve a precision convergence. The fact that the ShortPathHop, ShortPathDist and GeomRout have exactly the same level of battery consumption can be explained by the definition of this protocols, that choose the sink once the latter is in the coverage area of the selector node.

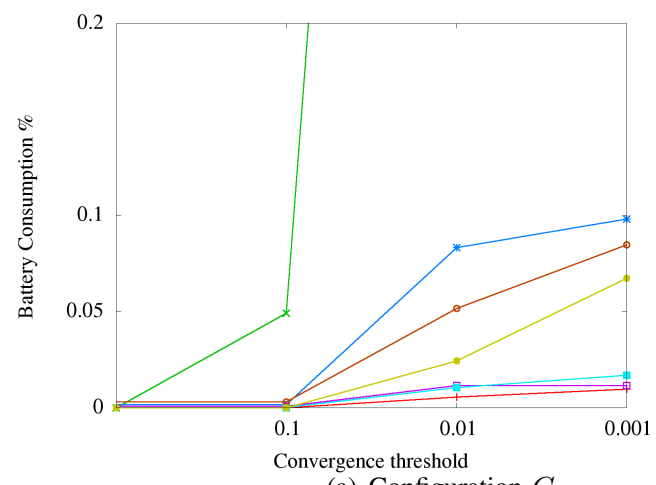

BroadcastRout — ProbaRout $\longrightarrow *$ GeomRout —* MultiPathRout $\longrightarrow$ ShortPathDist ArbitraryRout $\longrightarrow$

(a) Configuration $C_{1}$

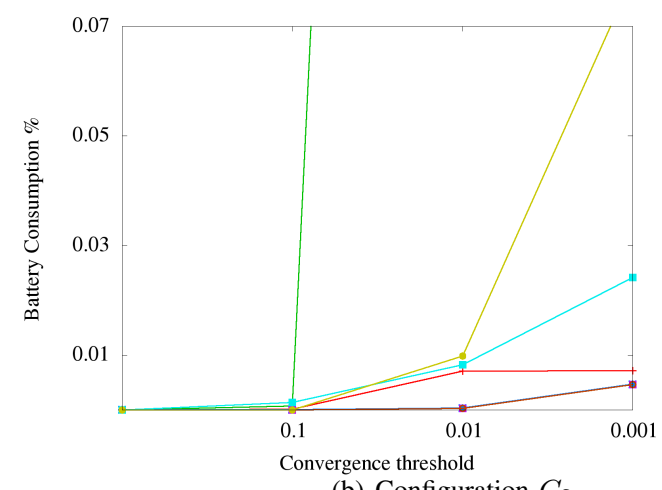

BroadcastRout — ProbaRout $\longrightarrow x$ GeomRout $\longrightarrow$ MultiPathRout ShortPathDist ArbitraryRout

(b) Configuration $C_{2}$

Fig. 2. Battery Consumption of optimization step

2) Optimization duration: To make the network operational as soon as possible, one of the important elements to reduce is the time that the system takes to be functional. What is meant by functional is the termination of optimization steps. Thus, Figure 3 depicts the optimization duration for $C_{1}$ and $C_{2}$ configurations, respectively. First, we can see that with $C_{1}$ configuration, once we change the routing protocol, the optimization duration changes also and the difference is not the slightest. In Figure 3(a), BroadcastRout protocol remains the most efficient and have the lowest optimization duration. Similarly, ProbaRout routing protocol remains the highest claimant in terms of optimization duration $(3333.479 \mathrm{Min})$. For the second configuration, we can observe that the ShortPathHop, ShortPathDist and GeomRout have the lowest optimization 
duration. However, if more precision than $10^{-2}$ is required, these latter give way to the BroadcastRout strategy. The figure shows also that ProbaRout protocol have the biggest optimization duration to a value of 1755.19 minutes.

This differences, whether in the first or in the second configuration, is directly related to the required number of iterations to gain a precision (with respect to $q_{i}$ ).
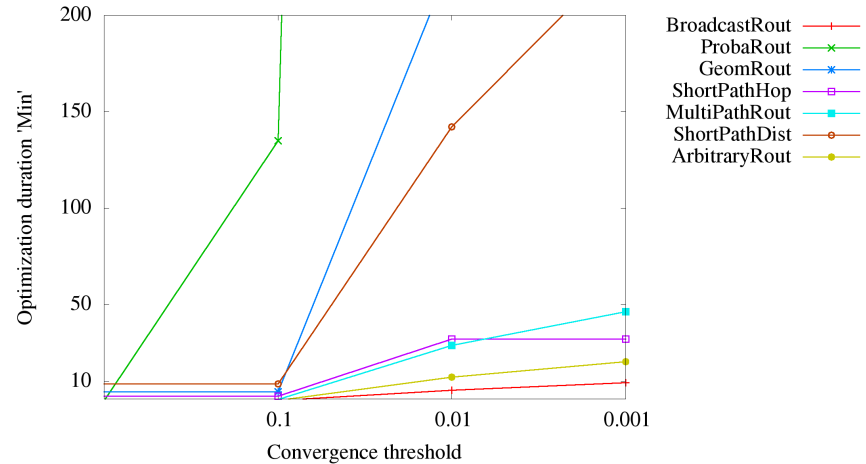

(a) Configuration $C_{1}$

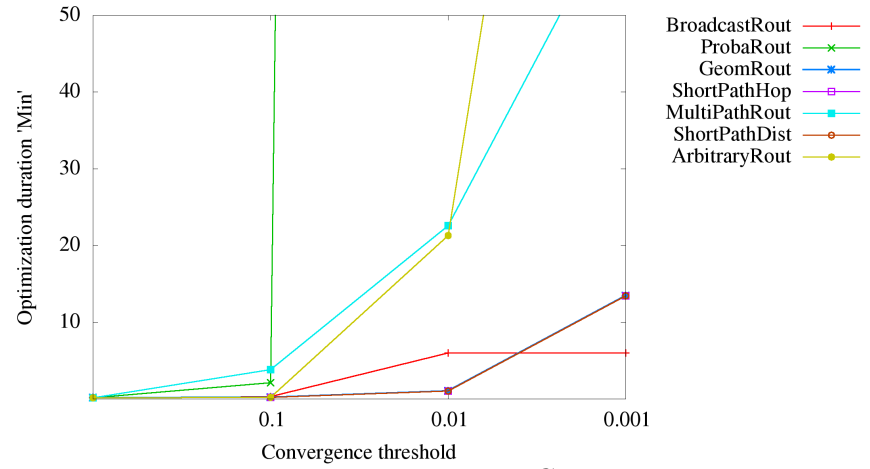

(b) Configuration $C_{2}$

Fig. 3. Activity duration

3) Network lifetime comparison: Let us focus now on the main objective of this work, which is the study of the impact of the chosen intermediate nodes for data routing on the network lifetime. We first recall that in our context, we consider critical applications on which the depletion of only one node entails the depletion of the whole network. Figure 4 presents the improvement of the lowest node lifetime (that directly affects the whole network lifetime), in each routing protocol for the $C_{1}$ and $C_{2}$ configurations. The two subfigures show that contrary to the battery consumption and optimization duration parameters, here the BroadcastRout routing protocol presents the lowest improvement of node's lifetime with an improvement of 2.79 and 1.59 times, respectively, compared to the initial node lifetime. This can be explained by the fact that in this strategy, each node must not only broadcasts its own data, but also broadcasts the multiple copies of the incoming data through its outgoing links. While the ShortPathDist for $C_{1}$ augmented with ShortPathHop and GeomRout for $C_{2}$ have the highest improvement of node's lifetime that was evaluated by 7.10 and 10.25 times, respectively, compared to the initial node lifetime. This can be explained by the fact that the distance between each node and its destination affect the transmission power consumption and thus the node lifetime.
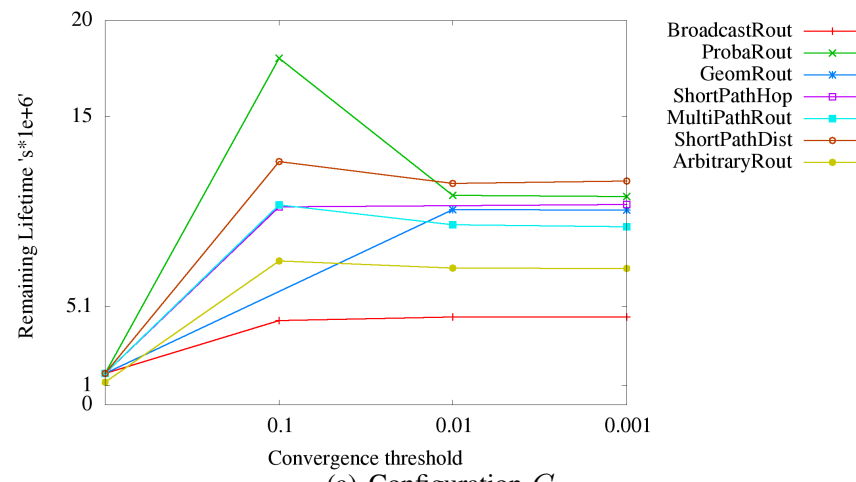
ProbaRout $\longrightarrow x$ GeomRout — ShortPathHop $\square$ MultiPathRout - $\longrightarrow$ ArbitraryRout $\longrightarrow$

(a) Configuration $C_{1}$

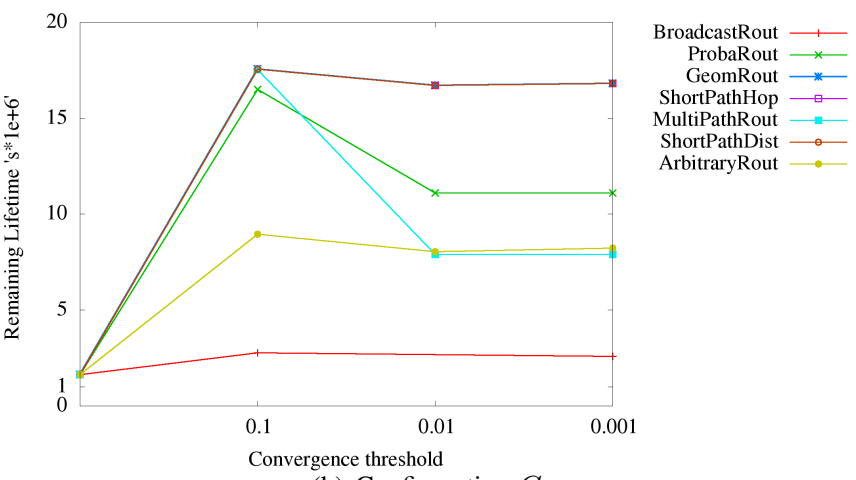

(b) Configuration $C_{2}$

Fig. 4. Remaining Lifetime

Let us now study the whole network lifetime. We remind that we have to minimize the inverse of the network lifetime, as recalled in section III-C. Thus, the lowest the common variable $q$ is, the longest the network lifetime is. Figure 5 depicts:

- the convergence of the different routing protocols to a common variable $q$,

- and the network lifetime using each of these protocols

For the $C_{1}$ configuration we can see that the ProbaRout protocol ensures the maximum network lifetime, while the MultiPathRout and ArbitraryRout have the lowest network lifetime, even compared to the BroadcastRout routing protocol. This slight difference can be explained by the fact that even if the BroadcastRout strategy floods the network by messages, this latter require much less iterations to converge to a common variable, allowing it to recover its huge requirement in term of transmission and reception power consumption. Contrariwise, in Figure 5(b), BroadcastRout presents the lowest network lifetime, that was expected, as in the second configuration, almost all the nodes are in the coverage area of each other. While the ShortPathDist, ShortPathHop and GeomRout routing protocols ensure the maximum network lifetime, since they need only one path to reach their destination, namely, the sink.

\section{DiscUSSIONS}

From the analyzes performed in the previous section, and based only on the first configuration $C_{1}$, we can easily say that the ProbaRout is the most appropriate routing protocol 

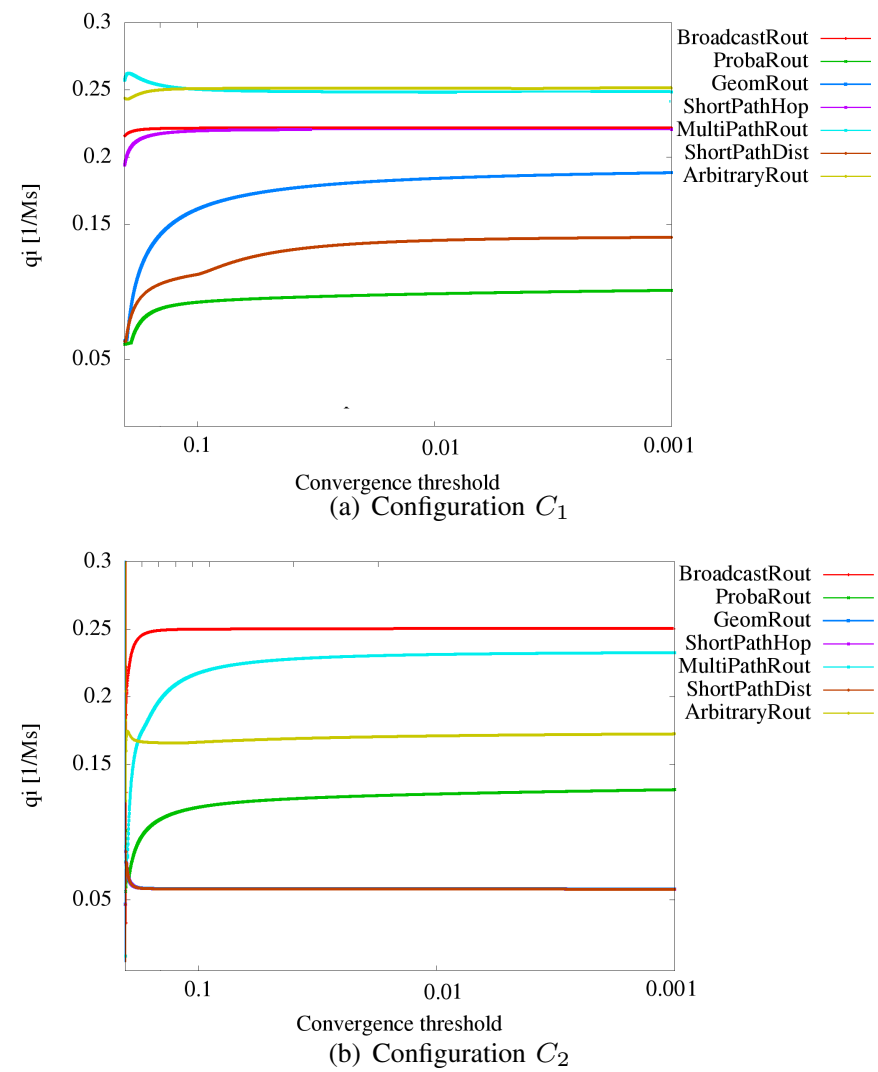

Fig. 5. Lifetime inverse $q=1 / T_{\text {net }}$

to transmit data in WMSNs, since the main objective is to maximize the network lifetime. However, taking into account the battery consumption and activity duration, this protocol presents the worst performance values.

On the other hand, once the configuration changes, the results also change instantly, and the difference is not the slightest (as shown in Figure 5(a) and Figure 5(b)).

Although the choice of the routing protocol depends on the application requirements, we can conclude that the ShortPathDist and GeomRout present in average a good results in the both two configurations. More precisely, they present a good percentage of battery consumption and an acceptable activity duration while ensuring a good level of network lifetime maximization. However, the ShortPathDist protocol is more efficient in the first configuration.

\section{CONCLUSIONS AND FUTURE WORK}

In this paper, we evaluated through simulation several routing protocols in WMSNs, through two network configurations. All the routing protocols were implemented as an add on to a fully distributed solution presented in a previous work [10], through MiXiM frame work, that was integrated in Omnet++ simulator. Simulations allowed us to compare the impact of each of these protocols on the network lifetime. In addition, the results show that, the choice of the forwarding nodes, affects enormously this latter.
For this reason the routing should be, without any doubt, considered and included in the optimization model to maximize the network lifetime. In our future work, we plan to integrate the routing optimization in an analytic model [10]. We plan thus to solve such a new problem in a fully distributed manner.

\section{ACKNOWLEDGEMENTS}

This article is partially funded by the Labex ACTION program (ANR-11-LABX-01-01 contract) and the FrancheComté regional council.

\section{REFERENCES}

[1] S. Boyd and L. Vandenberghe. Convex optimization. Cambridge university press, 2004.

[2] L. Cobo, A. Quintero, and S. Pierre. Ant-based routing for wireless multimedia sensor networks using multiple qos metrics. Computer networks, 54(17):2991-3010, 2010.

[3] D. do Rosário, R. Costa, H. Paraense, K. Machado, E. Cerqueira, and T. Braun. A smart multi-hop hierarchical routing protocol for efficient video communication over wireless multimedia sensor networks. In 2012 IEEE International Conference on Communications (ICC), pages 6530-6534. IEEE, 2012.

[4] W.-c. Feng, E. Kaiser, W. C. Feng, and M. L. Baillif. Panoptes: scalable low-power video sensor networking technologies. ACM Transactions on Multimedia Computing, Communications, and Applications (TOMM), 1(2):151-167, 2005.

[5] E. Gürses, Y. Lin, and R. Boutaba. Distributed quality-lifetime maximization in wireless video sensor networks. In Communications, 2009. ICC'09. IEEE International Conference on, pages 1-6. IEEE, 2009.

[6] Z. Hamid, F. B. Hussain, and J.-Y. Pyun. Delay and link utilization aware routing protocol for wireless multimedia sensor networks. Multimedia Tools and Applications, pages 1-22, 2015.

[7] Y. He, I. Lee, and L. Guan. Distributed algorithms for network lifetime maximization in wireless visual sensor networks. Circuits and Systems for Video Technology, IEEE Transactions on, 19(5):704-718, 2009.

[8] Z. He and D. Wu. Resource allocation and performance analysis of wireless video sensors. Electrical and Computer Engineering publications $(M U), 2006$

[9] Z. He and D. Wu. Resource allocation and performance analysis of wireless video sensors. Electrical and Computer Engineering publications (MU), 2006.

[10] N. Khernane, J.-F. Couchot, and A. Mostefaoui. Maximizing network lifetime in wireless video sensor networks under quality constraints. In Proceedings of the 14th ACM International Symposium on Mobility Management and Wireless Access. ACM, 2016.

[11] A. Köpke, M. Swigulski, K. Wessel, D. Willkomm, P. Haneveld, T. E. Parker, O. W. Visser, H. S. Lichte, and S. Valentin. Simulating wireless and mobile networks in omnet++ the mixim vision. In Proceedings of the 1st international conference on Simulation tools and techniques for communications, networks and systems \& workshops, page 71. ICST (Institute for Computer Sciences, Social-Informatics and Telecommunications Engineering), 2008.

[12] C. Li, J. Zou, H. Xiong, and C. W. Chen. Joint coding/routing optimization for distributed video sources in wireless visual sensor networks. IEEE Transactions on Circuits and Systems for Video Technology, 21(2):141-155, 2011.

[13] K. Lin, J. J. Rodrigues, H. Ge, N. Xiong, and X. Liang. Energy efficiency qos assurance routing in wireless multimedia sensor networks. IEEE Systems Journal, 5(4):495-505, 2011.

[14] N. Magaia, N. Horta, R. Neves, P. R. Pereira, and M. Correia. A multiobjective routing algorithm for wireless multimedia sensor networks. Applied Soft Computing, 30:104-112, 2015.

[15] D. P. Palomar and M. Chiang. A tutorial on decomposition methods for network utility maximization. Selected Areas in Communications, IEEE Journal on, 24(8):1439-1451, 2006.

[16] A. Varga et al. The omnet++ discrete event simulation system. In Proceedings of the European simulation multiconference (ESM2001), volume 9, S 185, page 65. sn, 2001. 